\title{
Cardiovascular Outcomes Among Patients with Type 2 Diabetes Newly Initiated on Sodium-Glucose Cotransporter-2 Inhibitors, Glucagon-Like Peptide-1 Receptor Agonists, and Other Antidiabetic Medications
}

\author{
Elmor D. Pineda, PharmD, MS; I-Chia Liao, MPH; Paul J. Godley, PharmD; \\ Jeffrey B. Michel, MD; and Karen L. Rascati, PhD
}

\begin{abstract}
BACKGROUND: Cardiovascular disease (CVD) remains the most prevalent cause of morbidity and mortality in patients with type 2 diabetes (T2D) and is a primary driver for health care costs associated with diabetes management. Sodium-glucose cotransporter-2 inhibitors (SGLT-2is) and glucagonlike peptide-1 receptor agonists (GLP-1 RAs) have demonstrated significant reductions in cardiovascular endpoints in clinical trials compared with placebo. However, it is uncertain whether these findings can be applied to the broader T2D population because these trials specifically included high-risk patients with established CVD.
\end{abstract}

OBJECTIVE: To evaluate and compare cardiovascular outcomes among adults with T2D newly initiated on SGLT-2is, GLP-1 RAs, and other antidiabetic medications (OADMs) in a real-world setting.

METHODS: This retrospective new-user cohort study used administrative claims and electronic health record data from an integrated delivery network in Texas. Patients aged $\geq 18$ years with T2D and $\geq 1$ prescription claim for an SGLT-2i, a GLP-1 RA, or an oADM filled between April 2013 and December 2018 were included. Patients were divided into three 1:1 propensity-matched groups according to index medication identified. Primary outcomes were heart failure hospitalization and a composite endpoint of myocardial infarction, stroke, unstable angina, or coronary revascularization. Cox proportional hazards regression was used to compare cumulative incidence of all outcome variables.

RESULTS: Among 9,477 patients, 1,134 were initiated on SGLT-2is, 1,072 on GLP-1 RAs, and 7,271 on oADMs. Patients initiating SGLT-2is versus 0ADMs had significantly lower risk of the composite endpoint $(\mathrm{HR}=0.64,95 \%$ $\mathrm{Cl}=0.46-0.90)$, heart failure hospitalization $(\mathrm{HR}=0.56,95 \% \mathrm{Cl}=0.39-0.81)$, and unstable angina requiring hospitalization $(\mathrm{HR}=0.56,95 \% \mathrm{Cl}=0.39$ 0.81). Patients initiating GLP-1 RAs compared with oADMs had significantly lower risk of the composite endpoint $(\mathrm{HR}=0.71,95 \% \mathrm{Cl}=0.52-0.98)$ and unstable angina requiring hospitalization $(\mathrm{HR}=0.60,95 \% \mathrm{Cl}=0.41-0.86)$. No differences in cardiovascular outcomes were found between SGLT-2is and GLP-1 RAs.

CONCLUSIONS: Both SGLT-2is and GLP-1 RAs showed significant reductions in the composite outcome and unstable angina requiring hospitalization versus oADMs. However, only SGLT-2is were associated with a lower risk for heart failure hospitalizations. Nevertheless, cardiovascular outcomes were similar between SGLT-2is and GLP-1 RAs.

J Manag Care Spec Pharm. 2020;26(5):610-18

Copyright $\odot 2020$, Academy of Managed Care Pharmacy. All rights reserved.

\section{What is already known about this subject}

Cardiovascular disease (CVD) remains the most prevalent cause of morbidity and mortality in patients with type 2 diabetes (T2D). Novel antidiabetic agents, namely sodium-glucose cotransporter-2 inhibitors (SGLT-2is) and glucagon-like peptide-1 receptor agonists (GLP-1 RAs), have demonstrated benefits in reducing cardiovascular event risk in patients with T2D with established CVD.

The real-world comparative reduction in cardiovascular risk associated with the use of SGLT-2is or GLP-1 RAs compared with other antidiabetic medications (oADMs) as well as the comparative effects between the 2 classes in routine clinical practice within the broader T2D population with or without CVD remain uncertain

\section{What this study adds}

In a population of adult patients with T2D with or without CVD, initiating SGLT-2is or GLP-1 RAs resulted in significantly reduced risk of cardiovascular events compared with initiating oADMs.

Patients initiating SGLT-2is compared with those initiating GLP-1 RAs had no difference in the risk of cardiovascular events.

This study provides real-world evidence for patients, payers, and providers to consider the selection of these novel antidiabetic agents with demonstrated cardiovascular benefits over other agents regardless of CVD status.

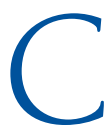
ardiovascular disease (CVD) remains the most prevalent cause of morbidity and mortality in patients with T2D. ${ }^{1}$ Approximately $32.2 \%$ of all patients with T2D have CVD, and roughly two thirds of deaths in this population are cardiovascular related.

Patients with T2D have a disproportionately 2- to 4-fold higher risk for atherosclerotic disease and develop CVD approximately 15 years earlier than their nondiabetic counterparts. ${ }^{2,3}$ A majority of this excess risk can be attributed to the high prevalence of additional underlying risk factors that are widely present in this population, including hypertension, dyslipidemia, obesity, sedentary lifestyle, chronic kidney 
disease (CKD), and smoking. Thus, the link between T2D and CVD is multifactorial, and control of blood glucose alone does not necessarily eliminate cardiovascular risk..$^{1,3}$

Joint management of T2D and CVD is imperative in lowering the risk of cardiovascular events, including myocardial infarction (MI), coronary heart disease, ischemic stroke, and heart failure hospitalizations. CVD has also been identified as 1 of the primary drivers for medical costs for diabetes and accounts for one quarter to one half of all direct costs. ${ }^{4-6}$ Adults with CVD and diabetes incur an additional $\$ 3,400$ to $\$ 9,700$ per patient per year in health care costs compared with adults with only diabetes. ${ }^{6}$ In 2017 , approximately $\$ 37.3$ billion in cardiovascular-related spending was associated with diabetes in the United States alone. ${ }^{4}$

Evidence of cardiovascular benefit with novel antihyperglycemic agents has created a paradigm shift in T2D management from focusing solely on glycemic targets to incorporating CVD risk reduction as well. Consequently, the American Diabetes Association and the American Association of Clinical Endocrinologists have updated their guidelines to incorporate consideration of atherosclerotic CVD for pharmacotherapy given the results of cardiovascular outcome trials (CVOTs) that suggest sodium glucose cotransporter-2 inhibitors (SGLT-2is) and glucagon-like peptide-1 receptor agonists (GLP-1 RAs) may decrease the risk of cardiovascular events and mortality through mechanisms beyond those related to their glycemic effects. Although lifestyle modifications and metformin are still recommended as initial therapy, agents with evidence of cardiovascular risk reduction, specifically empagliflozin, canagliflozin, liraglutide, and semaglutide, are recommended in patients with atherosclerotic CVD for secondary prevention. In March 2019, the American College of Cardiology (ACC) and the American Heart Association (AHA) released the 2019 Primary Prevention in Cardiovascular Disease guidelines, which outline strategies focused on primary prevention of CVD, including a recommendation for initiating an SGLT-2i or a GLP-1 RA as second-line or adjunct treatment to lifestyle modifications and metformin in adults with T2D, although, it received a weak level IIb recommendation. Nonetheless, this new guideline does not single out any preferred agent within each class, but rather provides the recommendation across their entirety.

Previous studies have attempted to investigate cardiovascular outcomes associated with the use of these agents. CVDREAL, a multinational industry-sponsored study, found that patients initiating SGLT-2is had lower risk of heart failure hospitalizations and all-cause death compared with those initiating other antidiabetic medications (oADMs), supporting a potential class effect among these agents. ${ }^{8}$ However, other relevant cardiovascular-related outcomes, such as MI, stroke, unstable angina, or coronary revascularizations were not included. In addition, no comparisons between SGLT-2is and specific classes were conducted. Therefore, conclusions regarding the comparative cardiovascular outcomes between SGLT-2is and GLP-1 RAs could not be ascertained. In CVD-REAL 2, SGLT-2is were associated with lower risk of MI and stroke compared with oADMs. ${ }^{9}$ Although the investigators improved their methods by including MI and stroke as endpoints, comparisons between SGLT-2is and specific classes were not performed. In addition, this study was limited to populations outside of the United States. Results from a similar study of commercially insured patients showed that canagliflozin was associated with a lower risk of heart failure hospitalizations; however, no differences were found in the risk of a composite of MI or stroke, an expanded composite of the primary composite endpoint, unstable angina, or coronary revascularization, or any of the individual components of the composite endpoints. ${ }^{10}$ This study did compare outcomes between an SGLT-2i and GLP-1 RAs; however, it was limited to a single agent without inclusion of other agents within the SGLT-2i class. Furthermore, findings appear to be inconsistent with prior studies regarding the association of lower MI and stroke risk across the SGLT-2i drug class.

Currently, the real-world comparative reduction in cardiovascular risk associated with the use of SGLT-2is or GLP-1 RAs compared with oADMs, as well as the comparative effects between the 2 classes in routine clinical practice remain uncertain. In addition, although SGLT-2is and GLP-1 RAs have demonstrated cardiovascular benefits compared with placebo, no direct comparisons have been made between SGLT-2is and GLP-1 RAs. As current interest has shifted from focusing solely on glycemic targets to incorporating cardiovascular outcomes when evaluating the effectiveness of antidiabetic therapy, realworld evidence comparing differences in cardiovascular outcomes among SGLT-2is, GLP-1 RAs, and oADMs will be useful for well-informed clinical decision making for patients, payers, and providers.

The purpose of this study was to evaluate and compare cardiovascular outcomes among adults with T2D newly initiated on SGLT-2is, GLP-1 RAs, or oADMs from a large integrated delivery network.

\section{Methods}

\section{Data Source}

A population-based retrospective new-user cohort study was conducted using administrative claims and electronic health record (EHR) data from April 1, 2012, through December 31, 2018. Patient-level data were extracted from the Virtual Data Warehouse, which houses pharmacy and medical claims for the Scott and White Health Plan (SWHP), and from EHR data from the Baylor Scott and White Health (BSWH) System. BSWH is an integrated delivery network, which includes 48 acute care hospitals, $>900$ patient care sites, and SWHP covers $>415,000$ commercial, Medicare, and Medicaid lives, geographically located within the central and northern Texas regions. 
Unique patient-level identifiers were used to longitudinally link pharmacy and medical claims to patient enrollment and medical care data containing demographic information. Pharmacy claims provided details from all dispensed prescriptions, including drug name, National Drug Code number, prescription fill dates, quantity dispensed, and days supplied. Medical claims provided detailed information on inpatient and outpatient services, including encounter dates, place of service, procedure codes, and up to 5 International Classification of Diseases, Ninth Revision, Clinical Modification (ICD-9-CM) and Tenth Revision (ICD-10-CM) diagnosis codes per episode of care. This study was approved by the Baylor Scott $\&$ White Research Institute and the University of Texas institutional review boards following expedited review.

\section{Study Population}

Patients were included if they were aged $\geq 18$ years at the time of cohort entry and had a diagnosis of T2D; $\geq 12$ months of continuous health plan enrollment in the pre-index (baseline) period; and at least 1 prescription claim for SGLT-2i, GLP-1 RA, or oADM (metformin, dipeptidyl peptidase-4 inhibitors [DPP-4is], sulfonylureas, thiazolidinediones [TZDs], meglinitides, and insulin) filled between April 1, 2013, and December 31, 2018 (index period). The first prescription date for SGLT-2i, GLP-1 RA, or oADM during the index period was referred to as the index date. A diagnosis of T2D was defined as having at least 1 inpatient or outpatient medical claim with an ICD9-CM of 250.x0 or 250.x2 or ICD-10-CM of E11.

Only new users, defined as having a prescription claim for SGLT-2i, GLP-1 RA, or oADM with no prescription claim within the same drug class during the 12-month baseline period, were included in the study. The active-comparator, new-user study design was selected to mitigate the need to adjust for confounding due to pretreatment effects, provide the ability to capture outcome-related events occurring after the initial start of therapy, and reduce the potential for confounding by varying disease severity. ${ }^{11}$ Patients were divided into 3 groups using a hierarchical approach according to the index medication identified: SGLT-2i, GLP-1 RA, or oADM. Patients were first assessed for inclusion in either the SGLT-2i or GLP-1 RA group. The remaining patients were then considered for the oADM group. In cases where patients initiated more than 1 oADM on the day of cohort entry, an index medication was randomly selected. Patients were excluded in the SGLT-2i group if a prescription claim for GLP-1 RA was identified during the baseline period, to reduce probable carryover effects of prior competitor use. Consideration of excluding GLP-1 RA patients with previous history of SGLT-2i use was not necessary, because the first SGLT-2i marketed in the United States was not approved until March 29, 2013. Other exclusion criteria included a diagnosis of type 1 diabetes or gestational diabetes.

\section{Baseline Patient Characteristics and Study Outcomes}

Demographic and clinical characteristics were measured during the 12-month baseline period. Demographic variables included age at the time of index date, gender, and race. Clinical characteristics included hemoglobin Alc (Alc), comorbidities, and glucose-lowering and cardiovascular-related medication utilization. Comorbidities were identified within medical claims by ICD-9-CM/ICD-10-CM and Current Procedural Terminology codes. Relevant comorbidities included history of CVD, atrial fibrillation, hypertension, CKD, microvascular disease, coronary revascularization, dyslipidemia, and obesity. Charlson Comorbidity Index (CCI) scores were calculated for each patient using ICD-9-CM/ICD-10-CM diagnosis codes. ${ }^{12}$

Follow-up began on the day after index date. Patients were followed over a 1-year period until occurrence of a study event, treatment discontinuation, switch to a comparator, end of continuous health plan enrollment, or end of the study period, whichever came first. Treatment discontinuation was defined as $>60$-day persistence gap. Persistence was calculated as the number of days from the date of first prescription claim to the date of first occurrence of prescription fill gap. Primary outcomes were a composite endpoint composed of hospital admissions for MI, stroke, unstable angina, or coronary revascularization, as well as heart failure hospitalization as defined in Appendix A (available in online article). Secondary outcomes were the individual components of the primary composite endpoint.

\section{Statistical Analysis}

Descriptive statistics were performed on all baseline variables. Continuous variables were described in medians with interquartile range (IQR), and categorical variables were described in frequency with percentages. Wilcoxon rank-sum tests were used for continuous measures and chi-square tests for categorical measures to detect differences between groups. Three multivariable logistic regression models were used to estimate propensity scores and predict the probability of being initiated on SGLT-2i versus oADM, GLP-1 RA versus oADM, and SGLT-2i versus GLP-1 RA, controlling for age, gender, comorbidities, and prior antidiabetic and cardiovascular-related medication use.

Propensity scores, using a caliper width equal to 0.2 of the standard deviation of the logit of the propensity score, were used to 1:1 match patients using a pairwise approach and nearest neighbor greedy algorithm to adjust for confounders and balance baseline patient characteristics between treatment groups. Pairwise comparisons of patient demographics and clinical characteristics between SGLT-2is and oADMs, GLP-1 RAs and oADMs, and SGLT-2is and GLP-1 RAs were compared before and after matching. A standardized difference of $>10 \%$ was considered a significant imbalance between groups.

Incident event rates were described for each cohort as the number of first occurrence of the outcome divided by the total number of person-years at risk. Cumulative incidence 
Cardiovascular Outcomes Among Patients with Type 2 Diabetes Newly Initiated on Sodium-Glucose Cotransporter-2 Inhibitors, Glucagon-Like Peptide-1 Receptor Agonists, and Other Antidiabetic Medications

\section{FIGURE 1 Patient Selection Flowchart}

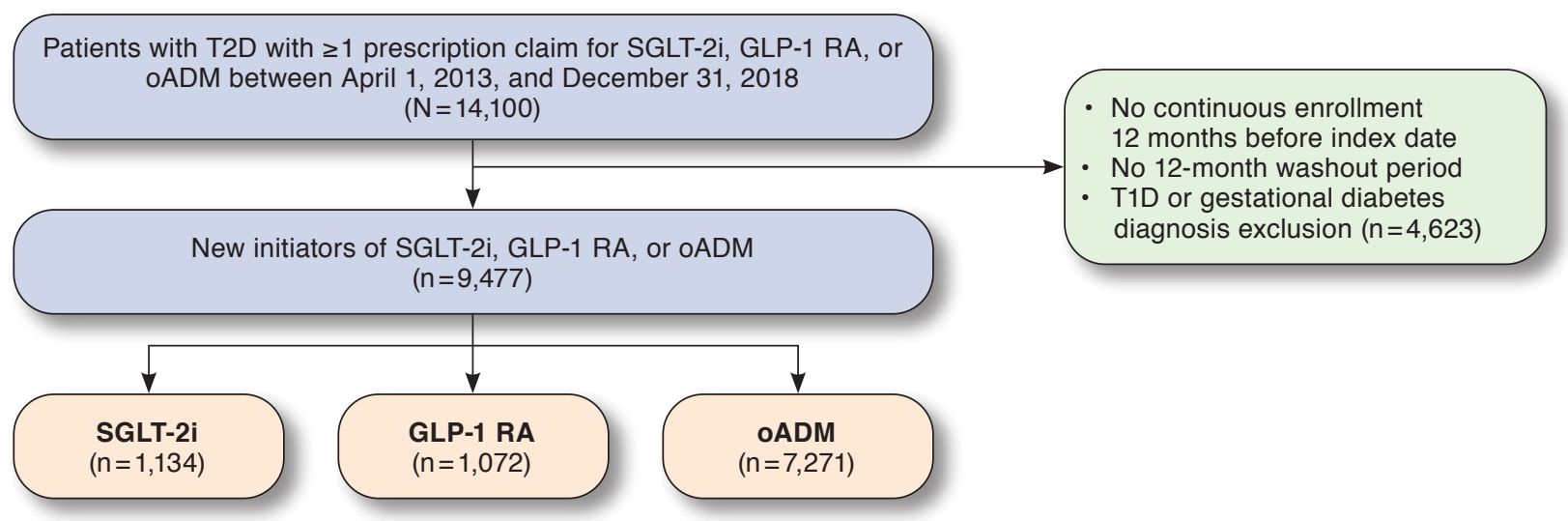

GLP-1 RA=glucagon-like peptide-1 receptor agonist; oADM = other antidiabetic medication; T1D=type 1 diabetes; T2D=type 2 diabetes; SGLT-2i=sodium-glucose cotransporter-2 inhibitor.

rates of cardiovascular outcomes were estimated using Cox proportional hazards regression. Hazard ratios (HR) with 95\% confidence intervals (CI) were reported for each outcome for all comparisons. Schoenfeld residuals tests were conducted to confirm that the proportional hazards assumption was not violated. All analyses were performed using SAS software package 9.4 (SAS Institute, Cary, NC). An alpha $<0.05$ was used as the criterion for statistical significance.

\section{Results}

From April 1, 2013, through December 31, 2018, a total of 14,100 patients with T2D who were new initiators of an SGLT-2i, a GLP-1 RA, or an oADM were identified. After applying study exclusions, 9,477 patients remained: 1,134 SGLT-2i initiators, 1,072 GLP-1 RA initiators, and 7,271 oADM initiators (Figure 1). Baseline patient characteristics before matching are described within each cohort and compared in Table 1. Before propensity score matching, patients initiated on SGLT-2is or GLP-1 RAs were younger and had lower prevalence of CVD; lower utilization of anticoagulants; higher prevalence of microvascular disease, dyslipidemia, and obesity; higher utilization of oral antidiabetic medications, insulin, statins, angiotensin-converting enzyme inhibitors, angiotensin II receptor blockers; and, for those with available values, higher Alc compared with those initiated on oADMs.

Compared with patients initiating GLP-1 RAs, those initiating SGLT-2is tended to be female; had lower prevalence of CKD and nephropathy; and had lower baseline utilization of sulfonylureas, insulin, and diuretics. However, they were more likely to have higher prevalence of dyslipidemia; had higher baseline utilization of metformin, DPP-4is, and TZDs; and had higher
Alc values. Table 2 shows comparisons of baseline characteristics within each cohort after matching. After propensity score matching, patient characteristics included in all 3 propensity score models were well balanced. There were 817 matched pairs in cohort 1 (SGLT-2i vs. oADM), 815 in cohort 2 (GLP-1 RA vs. oADM), and 947 in cohort 3 (SGLT-2i vs. GLP-1 RA). Distributions of index medications within each cohort are provided in Appendix B (available in online article).

Table 3 shows the associated risks for the primary and secondary outcomes in each pairwise propensity-matched cohort. Initiating SGLT-2is versus oADMs was associated with a lower risk of the composite endpoint ( $\mathrm{HR}=0.69,95 \% \mathrm{CI}=0.52-0.92$; $P=0.01$ ), with an incidence rate of 7.0 versus 8.5 events per 100 person-years, and a lower risk of heart failure hospitalizations ( $\mathrm{HR}=0.66,95 \% \mathrm{CI}=0.47-0.93 ; P=0.02)$ with an incidence rate of 4.7 versus 6.3 events per 100 person-years. Similarly, initiating GLP-1 RAs versus oADMs was associated with a lower risk of the composite endpoint ( $\mathrm{HR}=0.69,95 \% \mathrm{CI}=0.52-0.92$; $P=0.01$ ), with an incidence rate of 7.6 versus 8.2 events per 100 person-years; however, no difference was found in the risk for heart failure hospitalizations. When comparing patients initiating SGLTi-2is versus GLP-1 RAs, no significant differences were found in primary outcomes.

Compared with patients initiating oADMs, those initiating SGLT-2is or GLP-1 RAs had lower risk of unstable angina requiring hospitalization (4.7 vs. 6.4 events per 100 personyears $[\mathrm{HR}=0.58,95 \% \mathrm{CI}=0.41-0.82 ; P=0.002$, respectively $]$ and 5.3 vs. 6.4 events per 100 person-years $[\mathrm{HR}=0.58,95 \%$ $\mathrm{CI}=0.42-0.83 ; P=0.003$, respectively]). No significant differences in secondary outcomes were found between patients initiating SGLT-2is and GLP-1 RAs. In addition, there were no 
Cardiovascular Outcomes Among Patients with Type 2 Diabetes Newly Initiated on Sodium-Glucose Cotransporter-2 Inhibitors, Glucagon-Like Peptide-1 Receptor Agonists, and Other Antidiabetic Medications

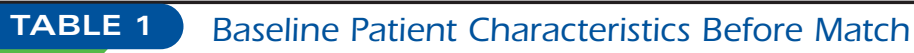

\begin{tabular}{|c|c|c|c|c|c|c|c|c|c|}
\hline \multirow[b]{2}{*}{ Variables } & \multicolumn{3}{|c|}{ Cohort 1} & \multicolumn{3}{|c|}{ Cohort 2} & \multicolumn{3}{|c|}{ Cohort 3} \\
\hline & $\begin{array}{l}\text { SGLT-2i } \\
(\mathrm{n}=1,134)\end{array}$ & $\begin{array}{l}\text { oADM } \\
(\mathbf{n}=7,271)\end{array}$ & $P$ Value & $\begin{array}{l}\text { GLP-1 RA } \\
(\mathrm{n}=1,072)\end{array}$ & $\begin{array}{c}\text { oADM } \\
(\mathrm{n}=7,271)\end{array}$ & $P$ Value & $\begin{array}{l}\text { SGLT-2i } \\
(\mathbf{n}=1,134)\end{array}$ & $\begin{array}{l}\text { GLP-1 RA } \\
(\mathrm{n}=1,072)\end{array}$ & $P$ Value \\
\hline \multicolumn{10}{|l|}{ Demographics } \\
\hline Age, years, median (IQR) & $55.0(14.0)$ & $60.0(20.0)$ & $<0.001$ & $55.0(15.0)$ & $60.0(20.0)$ & $<0.001$ & $55.0(14.0)$ & $55.0(15.0)$ & 0.27 \\
\hline \multicolumn{10}{|l|}{ Gender } \\
\hline Female & $554(48.9)$ & $3,501(48.2)$ & 0.66 & $640 \quad(57.9)$ & $3,501(48.2)$ & $<0.001$ & $554(48.9)$ & $640(59.7)$ & $<0.001$ \\
\hline \multicolumn{10}{|l|}{ Race } \\
\hline White & $442(39.0)$ & $3,863(53.1)$ & $<0.001$ & $433 \quad(40.4)$ & $3,863(53.1)$ & $<0.001$ & $442(39.0)$ & $433(40.4)$ & 0.50 \\
\hline Other & $106 \quad(9.4)$ & $891(12.3)$ & 0.005 & $\begin{array}{ll}06 & (9.9) \\
\end{array}$ & $891(12.3)$ & 0.03 & $106 \quad(9.4)$ & $106 \quad(9.9)$ & 0.67 \\
\hline Unknown & $586(51.7)$ & $2,517(34.6)$ & $<0.001$ & $533 \quad(49.7)$ & 2,517(34.6) & $<0.001$ & $586(51.7)$ & $533(49.7)$ & 0.36 \\
\hline \multicolumn{10}{|l|}{ Comorbidities } \\
\hline CCI score, median (IQR) & $2.0 \quad(2.0)$ & $1.0 \quad(2.0)$ & 0.7706 & \begin{tabular}{|ll}
2.0 & $(2.0)$ \\
\end{tabular} & $1.0 \quad(2.0)$ & 0.002 & $2.0 \quad(2.0)$ & $2.0 \quad(2.0)$ & 0.01 \\
\hline CVD history & $148(13.1)$ & $1,682(23.1)$ & $<0.001$ & $154.0 \quad(14.4)$ & $1,682(23.1)$ & $<0.001$ & $148(13.1)$ & $154.0(14.4)$ & 0.37 \\
\hline MI & $10 \quad(0.9)$ & $145 \quad(2.0)$ & $<0.001$ & $\begin{array}{ll}12.0 & (1.1) \\
\end{array}$ & $145 \quad(2.0)$ & 0.049 & $10 \quad(0.9)$ & $12.0 \quad(1.1)$ & 0.57 \\
\hline Stroke & $18 \quad(1.6)$ & $252 \quad(3.5)$ & $<0.001$ & $16.0 \quad(1.5)$ & $252 \quad(3.5)$ & 0.001 & $18 \quad(1.6)$ & $16.0 \quad(1.5)$ & 0.86 \\
\hline TIA & $17 \quad(1.5)$ & $136 \quad(1.9)$ & 0.38 & $15.0 \quad(1.4)$ & $136 \quad(1.9)$ & 0.28 & $17 \quad(1.5)$ & $15.0 \quad(1.4)$ & 0.84 \\
\hline Unstable angina & $82 \quad(7.2)$ & 987 (13.6) & $<0.001$ & $73.0 \quad(6.8)$ & 987 (13.6) & $<0.001$ & $82 \quad(7.2)$ & $73.0 \quad(6.8)$ & 0.70 \\
\hline Angina pectoris & $11 \quad(1.0)$ & $140 \quad(1.9)$ & 0.02 & $\begin{array}{ll}9.0 & (0.8) \\
\end{array}$ & $140 \quad(1.9)$ & 0.01 & $11 \quad(1.0)$ & $9.0 \quad(0.8)$ & 0.75 \\
\hline Heart failure & $47 \quad(4.1)$ & $\begin{array}{ll}702 & (9.7)\end{array}$ & $<0.001$ & $\begin{array}{ll}62.0 & (5.8) \\
\end{array}$ & $702 \quad(9.7)$ & $<0.001$ & $47 \quad(4.1)$ & $62.0 \quad(5.8)$ & 0.08 \\
\hline PAD & $13 \quad(1.2)$ & $179 \quad(2.5)$ & 0.006 & $18.0 \quad(1.7)$ & $179 \quad(2.5)$ & 0.12 & $13 \quad(1.2)$ & $18.0 \quad(1.7)$ & 0.29 \\
\hline Atrial fibrillation & $12 \quad(1.1)$ & $332 \quad(4.6)$ & $<0.001$ & $12.0 \quad(1.1)$ & $332 \quad(4.6)$ & $<0.001$ & $12 \quad(1.1)$ & $12.0 \quad(1.1)$ & 0.89 \\
\hline Hypertension & $898(79.2)$ & $5,690(78.3)$ & 0.48 & 875.0 (81.6) & $5,690(78.3)$ & 0.01 & $898(79.2)$ & $875.0(81.6)$ & 0.15 \\
\hline CKD & $48 \quad(4.2)$ & $609 \quad(8.4)$ & $<0.001$ & $(9.2)$ & $609 \quad(8.4)$ & 0.35 & $48 \quad(4.2)$ & $99.0 \quad(9.2)$ & $<0.001$ \\
\hline Microvascular disease & $649(57.2)$ & $2,813(38.7)$ & $<0.001$ & $648.0 \quad(60.5)$ & $2,813(38.7)$ & $<0.001$ & $649(57.2)$ & $648.0(60.5)$ & 0.13 \\
\hline Dyslipidemia & $934(82.4)$ & $5,041 \quad(69.3)$ & $<0.001$ & $838.0 \quad(78.2)$ & $5,041(69.3)$ & $<0.001$ & $934(82.4)$ & $838.0(78.2)$ & 0.01 \\
\hline Obesity & $508(44.8)$ & $2,746(37.8)$ & $<0.001$ & $616.0 \quad(57.5)$ & $2,746(37.8)$ & $<0.001$ & $508(44.8)$ & $616.0(57.5)$ & $<0.001$ \\
\hline \multicolumn{10}{|l|}{ Antidiabetic medications } \\
\hline $\begin{array}{l}\text { Antidiabetic medications, } \\
\mathrm{n} \text {, median (IQR) }\end{array}$ & $2.0 \quad(2.0)$ & $0.0 \quad(1.0)$ & $<0.001$ & $2.0 \quad(2.0)$ & $0.0 \quad(1.0)$ & $<0.001$ & $2.0 \quad(2.0)$ & $2.0 \quad(2.0)$ & $<0.001$ \\
\hline Metformin & $908(80.1)$ & $1,450(19.9)$ & $<0.001$ & $787.0 \quad(73.4)$ & $1,450(19.9)$ & $<0.001$ & $908(80.1)$ & $787.0(73.4)$ & $<0.001$ \\
\hline DPP-4i & $358(31.6)$ & $240 \quad(3.3)$ & $<0.001$ & $252.0(23.5)$ & $240 \quad(3.3)$ & $<0.001$ & $358(31.6)$ & $252.0(23.5)$ & $<0.001$ \\
\hline SU & $581(51.2)$ & $992(13.6)$ & $<0.001$ & $477.0 \quad(44.5)$ & 992 (13.6) & $<0.001$ & $581(51.2)$ & $477.0(44.5)$ & 0.002 \\
\hline TZD & $104 \quad(9.2)$ & $76 \quad(1.1)$ & $<0.001$ & $69.0 \quad(6.4)$ & $76 \quad(1.1)$ & $<0.001$ & $104 \quad(9.2)$ & $69.0 \quad(6.4)$ & 0.02 \\
\hline Meglinitides & $11 \quad(1.0)$ & $5 \quad(0.1)$ & $<0.001$ & $(0.8)$ & $5 \quad(0.1)$ & $<0.001$ & $11 \quad(1.0)$ & $8.0 \quad(0.8)$ & 0.57 \\
\hline Insulin & $370(32.6)$ & $484 \quad(6.7)$ & $<0.001$ & $442.0 \quad(41.2)$ & $484 \quad(6.7)$ & $<0.001$ & $370(32.6)$ & $442.0(41.2)$ & $<0.001$ \\
\hline \multicolumn{10}{|c|}{ Cardiovascular-related medications } \\
\hline ACEi & $561(49.5)$ & $2,706(37.2)$ & $<0.001$ & $511.0 \quad(47.7)$ & $2,706(37.2)$ & $<0.001$ & $561(49.5)$ & $511.0(47.7)$ & 0.40 \\
\hline ARB & $294(25.9)$ & $1,297(17.8)$ & $<0.001$ & $316.0(29.5)$ & 1,297 (17.8) & $<0.001$ & $294(25.9)$ & $316.0(29.5)$ & 0.06 \\
\hline Beta blocker & $305(26.9)$ & $2,267(31.2)$ & 0.004 & $324.0(30.2)$ & $2,267(31.2)$ & 0.53 & $305(26.9)$ & $324.0(30.2)$ & 0.08 \\
\hline $\mathrm{CCB}$ & $230(20.3)$ & $1,509(20.8)$ & 0.72 & $248.0(23.1)$ & $1,509(20.8)$ & 0.07 & $230(20.3)$ & $248.0(23.1)$ & 0.10 \\
\hline Thiazide diuretic & $361(31.8)$ & $2,002 \quad(27.5)$ & 0.003 & $396.0 \quad(36.9)$ & $2,002(27.5)$ & $<0.001$ & $361(31.8)$ & $396.0(36.9)$ & 0.01 \\
\hline Loop diuretic & $85 \quad(7.5)$ & $812(11.2)$ & 0.002 & $110.0(10.3)$ & $812(11.2)$ & 0.38 & $85 \quad(7.5)$ & $110.0(10.3)$ & 0.02 \\
\hline Nitrate & $45 \quad(4.0)$ & $347 \quad(4.8)$ & 0.23 & (4.9) & $347 \quad(4.8)$ & 0.91 & $45 \quad(4.0)$ & $52.0 \quad(4.9)$ & 0.31 \\
\hline Anticoagulant & $29 \quad(2.6)$ & $333 \quad(4.6)$ & 0.002 & $24.0 \quad(2.2)$ & $333 \quad(4.6)$ & $<0.001$ & $29 \quad(2.6)$ & $24.0 \quad(2.2)$ & 0.63 \\
\hline Antiplatelet & $82 \quad(7.2)$ & $453 \quad(6.2)$ & 0.20 & $85.0 \quad(7.9)$ & $453 \quad(6.2)$ & 0.04 & $82 \quad(7.2)$ & $85.0 \quad(7.9)$ & 0.54 \\
\hline Statin & $786(69.3)$ & $3,447 \quad(47.4)$ & $<0.001$ & $708.0 \quad(66.0)$ & $3,447 \quad(47.4)$ & $<0.001$ & $786(69.3)$ & $708.0(66.0)$ & 0.10 \\
\hline \multicolumn{10}{|l|}{ Alc } \\
\hline Alc, median (IQR) & $8.8 \quad(1.9)$ & $7.6 \quad(2.1)$ & $<0.001$ & (1.9) & $7.6 \quad(2.1)$ & $<0.001$ & $8.8 \quad(1.9)$ & $8.4 \quad(1.9)$ & 0.02 \\
\hline $\begin{array}{l}\text { Patients with Alc results } \\
\text { available }\end{array}$ & $334(29.5)$ & $2,564(36.3)$ & $<0.001$ & $304.0 \quad(28.4)$ & $2,564(35.3)$ & $<0.001$ & $334(29.5)$ & $304.0(28.4)$ & 0.57 \\
\hline
\end{tabular}

Note: Data are reported as $n(\%)$ unless otherwise stated. $P$ values in bold indicated statistical significance.

$A 1 c=$ hemoglobin $A 1 c ; A C E i=$ angiotensin-converting enzyme inhibitor; $A R B=$ angiotensin II receptor blocker; $C C B=$ calcium channel blocker; $C C I=C h a r l s o n$ Comorbidity Index; $C K D=$ chronic kidney disease; $C V D=$ cardiovascular disease; $D P P-4 i=$ dipeptidyl peptidase-4 inhibitor; GLP-1 RA=glucagon-like peptide-1 receptor agonist;

$I Q R=$ interquartile range; $O A D M=$ other antidiabetic medication; $M I=$ myocardial infarction; $P A D=$ peripheral artery disease; SGLT- $2 i=$ sodium-glucose cotransporter -2 inhibitor; $S U=$ sulfonylurea; $T I A=$ transient ischemic attack; $T Z D=$ thiazolidinedione 
Cardiovascular Outcomes Among Patients with Type 2 Diabetes Newly Initiated on Sodium-Glucose Cotransporter-2 Inhibitors, Glucagon-Like Peptide-1 Receptor Agonists, and Other Antidiabetic Medications

TABLE 2

Baseline Patient Characteristics After Match

\begin{tabular}{|c|c|c|c|c|c|c|c|c|c|}
\hline \multirow[b]{2}{*}{ Variables } & \multicolumn{3}{|c|}{ Cohort 1} & \multicolumn{3}{|c|}{ Cohort 2} & \multicolumn{3}{|c|}{ Cohort 3} \\
\hline & $\begin{array}{l}\text { SGLT-2i } \\
(\mathrm{n}=815)\end{array}$ & $\begin{array}{c}\text { oADM } \\
(\mathrm{n}=815)\end{array}$ & $\begin{array}{c}\text { STD } \\
\text { Difference }\end{array}$ & $\begin{array}{c}\text { GLP-1 RA } \\
(\mathrm{n}=817)\end{array}$ & $\begin{array}{c}\text { oADM } \\
(\mathrm{n}=817)\end{array}$ & $\begin{array}{c}\text { STD } \\
\text { Difference }\end{array}$ & $\begin{array}{l}\text { SGLT-2i } \\
(\mathrm{n}=947)\end{array}$ & $\begin{array}{l}\text { GLP-1 RA } \\
(\mathrm{n}=947)\end{array}$ & $\begin{array}{c}\text { STD } \\
\text { Difference }\end{array}$ \\
\hline \multicolumn{10}{|l|}{ Demographics } \\
\hline Age, years, median (IQR) & $56.0(14.0)$ & $58.0(18.0)$ & -0.1 & $55.0(14.0)$ & $56.0(16.0)$ & 0.1 & $55.0(14.0)$ & $55.0(15.0)$ & 0.1 \\
\hline \multicolumn{10}{|l|}{ Gender } \\
\hline Female & 48.8 & 48.8 & 0.0 & 58.0 & 58.0 & 0.0 & 57.7 & 57.7 & 0.0 \\
\hline \multicolumn{10}{|l|}{ Comorbidities } \\
\hline CCI, median (IQR) & $2.0(2.0)$ & $2.0(2.0)$ & -0.1 & $2.0(2.0)$ & $2.0(2.0)$ & 0.0 & $2.0(2.0)$ & $2.0(15.0)$ & 0.0 \\
\hline History of CVD & 14.8 & 17.9 & 0.0 & 15.3 & 16.3 & 0.0 & 12.0 & 12.8 & 0.0 \\
\hline MI & 0.9 & 0.9 & 0.1 & 1.4 & 1.0 & 0.0 & 0.8 & 1.2 & 0.0 \\
\hline Stroke & 2.0 & 2.1 & 0.0 & 1.2 & 1.4 & 0.0 & 1.4 & 1.5 & 0.0 \\
\hline Unstable angina & 8.5 & 10.4 & 0.1 & 8.1 & 9.0 & 0.0 & 6.3 & 7.0 & 0.0 \\
\hline Heart failure & 4.5 & 5.8 & 0.1 & 6.0 & 6.8 & 0.0 & 4.4 & 4.1 & 0.0 \\
\hline PAD & 1.4 & 1.7 & 0.0 & 1.8 & 1.7 & 0.0 & 1.2 & 1.3 & 0.0 \\
\hline Atrial fibrillation & 1.4 & 1.2 & 0.0 & 1.5 & 1.6 & 0.01 & 1.0 & 1.1 & 0.0 \\
\hline Hypertension & 79.8 & 80.9 & 0.0 & 81.0 & 80.3 & 0.0 & 79.0 & 80.3 & 0.0 \\
\hline CKD & 4.8 & 6.5 & 0.1 & 9.1 & 9.5 & 0.0 & 4.5 & 4.9 & 0.0 \\
\hline Microvascular disease & 51.7 & 49.7 & 0.0 & 55.0 & 50.0 & 0.1 & 58.4 & 58.3 & 0.0 \\
\hline Dyslipidemia & 81.2 & 82.2 & 0.1 & 76.6 & 76.9 & 0.0 & 80.6 & 79.0 & 0.0 \\
\hline Obesity & 48.6 & 46.9 & 0.0 & 58.2 & 58.7 & 0.0 & 54.6 & 59.1 & 0.1 \\
\hline \multicolumn{10}{|l|}{ Antidiabetic medications } \\
\hline $\begin{array}{l}\text { Number of antidiabetic } \\
\text { medications, median (IQR) }\end{array}$ & $2.0(1.0)$ & $2.0(1.0)$ & 0.0 & $2.0(1.0)$ & $2.0(1.0)$ & 0.0 & $2.0(2.0)$ & $2.0(2.0)$ & 0.1 \\
\hline Metformin & 73.0 & 74.4 & 0.0 & 66.8 & 70.4 & 0.1 & 78.8 & 77.5 & 0.0 \\
\hline DPP-4i & 22.2 & 18.6 & -0.1 & 18.4 & 16.3 & 0.1 & 29.7 & 25.5 & 0.1 \\
\hline SU & 44.2 & 44.9 & 0.0 & 38.8 & 38.3 & 0.0 & 49.7 & 47.4 & 0.1 \\
\hline TZD & 5.0 & 4.8 & 0.0 & 5.0 & 4.1 & 0.1 & 9.1 & 7.2 & 0.1 \\
\hline Insulin & 24.4 & 24.7 & 0.0 & 31.2 & 27.7 & 0.1 & 34.2 & 37.4 & 0.1 \\
\hline \multicolumn{10}{|c|}{ Cardiovascular-related medications } \\
\hline ACEi & 49.2 & 50.4 & 0.0 & 47.0 & 47.5 & 0.0 & 48.8 & 47.3 & 0.0 \\
\hline $\mathrm{ARB}$ & 25.3 & 25.0 & 0.0 & 27.4 & 27.4 & 0.0 & 26.4 & 27.8 & 0.0 \\
\hline Beta blocker & 20.9 & 22.4 & 0.1 & 31.3 & 31.2 & 0.0 & 26.6 & 28.1 & 0.0 \\
\hline CCB & 20.9 & 22.4 & 0.0 & 22.5 & 23.1 & 0.0 & 20.4 & 22.0 & 0.1 \\
\hline Thiazide diuretic & 32.9 & 32.3 & 0.0 & 36.3 & 34.2 & 0.0 & 33.2 & 34.6 & 0.0 \\
\hline Loop diuretic & 2.8 & 2.7 & 0.1 & 11.7 & 12.4 & 0.0 & 7.9 & 8.2 & 0.0 \\
\hline Anticoagulant & 2.8 & 2.7 & 0.0 & 2.7 & 2.3 & 0.0 & 2.2 & 2.0 & 0.0 \\
\hline Statin & 68.2 & 70.3 & 0.0 & 62.5 & 63.6 & 0.0 & 68.3 & 66.1 & 0.1 \\
\hline
\end{tabular}

Note: Data are reported as \% unless otherwise stated.

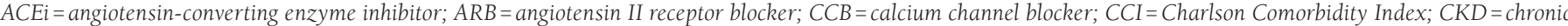
kidney disease; $C V D=$ cardiovascular disease; $D P P-4 i=$ dipeptidyl peptidase- 4 inhibitor; GLP-1 RA=glucagon-like peptide-1 receptor agonist; IQR=interquartile range; $O A D M=$ other antidiabetic medication; $M I=$ myocardial infarction; $P A D=$ peripheral artery disease; SGLT-2i=sodium-glucose cotransporter-2 inhibitor; $S T D=$ standardized $S U=$ sulfonylurea; $T I A=$ transient ischemic attack; $T Z D=$ thiazolidinedione

significant differences in the risks of MI, stroke, or coronary revascularization between SGLT-2is and oADMs or GLP-1 RAs and oADMs.

\section{Discussion}

To our knowledge, this is the first population-based, real-world study to evaluate the comparative effectiveness of initiating SGLT-2is, GLP-1 RAs, or oADMs on cardiovascular outcomes among a T2D population, a majority of whom had no previous history of CVD, within an integrated delivery network. In our institution, T2D patients initiating SGLT-2is and GLP-1 RAs were relatively similar. However, patients initiating GLP-1 RAs had higher prevalence of CKD and obesity at baseline, likely as a result of the contraindications and precautions of SGLT-2is in patients with renal impairment and the preference of GLP-1 RAs over SGLT-2is for minimizing weight gain or promoting weight loss. In addition, differences in baseline antidiabetic medication use were observed between these groups, with SGLT-2i patients having higher utilization of other oral agents. One reasonable explanation may be the relatively easier oral-to-oral conversion 
Cardiovascular Outcomes Among Patients with Type 2 Diabetes Newly Initiated on Sodium-Glucose Cotransporter-2 Inhibitors, Glucagon-Like Peptide-1 Receptor Agonists, and Other Antidiabetic Medications

\section{TABLE 3 Risk of Primary and Secondary Outcomes for Patients Within Each Pairwise Propensity Score} Matched Cohort

\begin{tabular}{|c|c|c|c|c|c|c|c|c|c|}
\hline & \multicolumn{3}{|c|}{ Cohort $1(n=817$ pairs $)$} & \multicolumn{3}{|c|}{ Cohort 2 ( $n=815$ pairs $)$} & \multicolumn{3}{|c|}{ Cohort 3 ( $n=947$ pairs $)$} \\
\hline & SGLT-2i & oADM & $P$ Value & GLP-1 RA & oADM & $P$ Value & SGLT-2i & GLP-1 RA & $P$ Value \\
\hline \multicolumn{10}{|l|}{ Primary outcomes } \\
\hline \multicolumn{10}{|c|}{ Cardiovascular composite endpoint } \\
\hline Follow-up, mean days, $\mathrm{n}$ & 262 & 277 & & 242 & 272 & & 265 & 246 & \\
\hline Incidence rate ${ }^{a}$ & 7.0 & 8.5 & & 7.6 & 8.2 & & 6.2 & 6.2 & \\
\hline Hazard ratio $(95 \% \mathrm{CI})$ & $0.64(0.46-0.90)$ & & 0.01 & $0.71(0.52-0.98)$ & & 0.04 & $1.00(0.69-1.44)$ & & 0.99 \\
\hline \multicolumn{10}{|l|}{ Heart failure hospitalization } \\
\hline Follow-up, mean days, $\mathrm{n}$ & 269 & 289 & & 244 & 281 & & 271 & 250 & \\
\hline Incidence rate ${ }^{a}$ & 4.7 & 6.3 & & 6.4 & 6.9 & & 4.1 & 4.9 & \\
\hline Hazard ratio $(95 \% \mathrm{CI})$ & $0.62(0.40-0.95)$ & & 0.03 & $0.80(0.54-1.17)$ & & 0.25 & $0.83(0.53-1.30)$ & & 0.41 \\
\hline \multicolumn{10}{|l|}{ Secondary outcomes } \\
\hline \multicolumn{10}{|c|}{ Unstable angina requiring hospitalization } \\
\hline Follow-up, mean days, $\mathrm{n}$ & 266 & 280 & & 245 & 274 & & 268 & 248 & \\
\hline Incidence rate ${ }^{\mathrm{a}}$ & 4.7 & 6.4 & & 5.3 & 6.4 & & 4.3 & 4.4 & \\
\hline Hazard ratio $(95 \% \mathrm{CI})$ & $0.56(0.39-0.81)$ & & 0.002 & $0.60(0.41-0.86)$ & & 0.006 & $0.97(0.64-1.48)$ & & 0.89 \\
\hline \multicolumn{10}{|l|}{ Myocardial infarction } \\
\hline Follow-up, mean days, $\mathrm{n}$ & 278 & 302 & & 255 & 296 & & 278 & 257 & \\
\hline Incidence rate $\mathrm{a}^{\mathrm{a}}$ & 1.2 & 1.0 & & 1.7 & 1.5 & & 1.3 & 1.4 & \\
\hline Hazard ratio $(95 \% \mathrm{CI})$ & $0.76(0.24-2.4)$ & & 0.64 & $1.29(0.47-3.57)$ & & 0.62 & $1.12(0.34-3.68)$ & & 0.85 \\
\hline \multicolumn{10}{|l|}{ Stroke } \\
\hline Follow-up, mean days, $\mathrm{n}$ & 275 & 300 & & 254 & 295 & & 277 & 256 & \\
\hline Incidence rate $\mathrm{a}^{\mathrm{a}}$ & 1.5 & 1.8 & & 1.2 & 1.2 & & 1.1 & 0.8 & \\
\hline Hazard ratio $(95 \% \mathrm{CI})$ & $0.95(0.42-2.16)$ & & 0.91 & $1.25(0.57-2.73)$ & & 0.58 & $0.87(0.38-1.97)$ & & 0.73 \\
\hline \multicolumn{10}{|l|}{ Coronary revascularization } \\
\hline Follow-up, mean days, $\mathrm{n}$ & 276 & 302 & & 255 & 297 & & 277 & 257 & \\
\hline Incidence rate ${ }^{\mathrm{a}}$ & 1.4 & 1.3 & & 1.5 & 1.2 & & 1.3 & 1.3 & \\
\hline Hazard ratio $(95 \% \mathrm{CI})$ & $2.18(0.66-7.24)$ & & 0.20 & $2.71(0.83-8.80)$ & & 0.10 & $1.05(0.38-2.89)$ & & 0.93 \\
\hline
\end{tabular}

with SGLT-2is, whereas the conversion from an oral agent to an injectable, such as a GLP-1 RA, may not be preferred by most patients. Insulin use was higher for patients initiating GLP-1 RAs compared with SGLT-2is, likely explained by the increasing popularity of combining both agents for their synergistic effects on glucose-lowering and opposing weight effects.

In contrast, patients initiating SGLT-2is and GLP-1 RAs compared with oADMs showed significant differences in baseline characteristic. These patients tended to be younger with lower prevalence of CVD but higher prevalence of microvascular disease. This is surprising because, based on our estimate of diabetes severity utilizing multiple proxy indicators, it would seem that most patients initiating oADMs had lower severity and may have been more recently diagnosed with T2D, given that $>50 \%$ of the oADM group were new initiators of metformin, had less antidiabetic medications at baseline, and had lower Alc values. However, it is important to note that $>60 \%$ of our patients had missing Alc values due to a large proportion of patients receiving tests from external facilities that do not directly report these values within the internal EHR system, which are therefore not captured within the structured dataset. Nevertheless, our method of propensity score matching each pairwise comparison group allowed for a similar distribution of measured covariates between each group.

Our findings showed that both SGLT-2is and GLP-1 RAs significantly reduced the risk of the composite endpoint comprised of MI, stroke, unstable angina, or coronary revascularization compared with oADMs by $36 \%$ and $29 \%$, respectively, over a 1-year follow-up period in a population of patients with $\mathrm{T} 2 \mathrm{D}$ and low prevalence of established CVD. The majority of completed CVOTs to date recruited high cardiovascular risk populations with almost all patients having established CVD, which is not representative of the general T2D population. In contrast, the majority of patients in our study $(>80 \%)$ had no history of CVD with $<9 \%$ having any history of heart failure.

Although it is difficult to compare our results with most of the CVOTs for these agents due to the lack of access to mortality data, we can at least compare data regarding nonfatal events. Our results showed that SGLT-2is reduced the risk of heart failure hospitalization by $38 \%$, which is in line with what 
was seen in EMPA-REG OUTCOME, CANVAS, and DECLARETIMI 58 at 35\%,33\%, and 27\%, respectively. ${ }^{13-15}$ This may provide some evidence in considering SGLT-2is early in the treatment decision algorithm for primary prevention of CVD, specifically for heart failure. For GLP-1 RAs, our results showed a nonsignificant reduction in heart failure hospitalization compared with oADMs, which is also consistent to what was seen in CVOTs. When comparing the cardiovascular event risk of both agents versus placebo, Kaplan-Meier curves appeared to separate early within the first few months for SGLT-2is, whereas the curves start to separate after about 12 months for GLP-1 RAs. ${ }^{16}$ This suggests that cardiovascular benefits for SGLT-2is are primarily driven by reductions in heart failure hospitalizations mediated by their positive hemodynamic effects, whereas those for GLP-1 RAs are primarily due to their effect on altering the progression of atherosclerosis, which is a relatively longer process. Consequently, a longer follow-up period, which was not attainable in our study, may have been needed to see these effects on reducing atherosclerotic CVD event risk for GLP-1 RAs, as well as for SGLT-2is.

The lack of differences in cardiovascular outcomes between SGLT-2is and GLP-1 RAs in our study suggests that both drug classes, in general, may be equally effective in reducing cardiovascular risk in the broad T2D population. Currently, clinical guidelines only provide recommendations for these drug classes to be used specifically for T2D patients with established CVD. Moreover, only select agents within each drug class-empagliflozin, canagliflozin, liraglutide, and semaglutide-are recommended. However, it seems that a new paradigm shift in the primary prevention of CVD may be underway as elucidated from the recent ACC/AHA guidelines for the Primary Prevention in Cardiovascular Disease. ${ }^{7}$ Additional evidence surrounding the broader use of these agents is expected to emerge in the upcoming years with investigations on the use of SGLT-2is in the absence of T2D, CVOT results that include a population of patients without CVD, as is the case with REWIND where only $31 \%$ reportedly had established CVD, as well as the first oral GLP-1 RA (semaglutide) expected to come to market.

\section{Limitations}

This study has several limitations. First, given the retrospective nature of the study, there is always a risk of residual unmeasured confounding that could not be entirely accounted for from the information available with single-system administrative claims and EHR data alone. Of 9,477 unique patients, only $45.7 \%$ had available Alc, preventing the use of Alc as a covariate in our propensity score models. Multiple imputation and missing indicator methods were considered possible solutions $^{17}$; however, it was unlikely the best approach without introducing significant bias, given that $>50 \%$ of the study population had missing values.
Second, we were unable to directly consider diabetes duration for each patient, which has been found to be positively correlated with cardiovascular event risk. ${ }^{18}$ Nonetheless, we were able to incorporate other related measures as proxies for evaluating diabetes severity, such as the presence of microvascular and macrovascular disease and the number of baseline antidiabetic agents.

Third, our data source did not allow us to investigate cardiovascular death as an outcome of interest, preventing the ability to compare our results to other studies that examined cardiovascular mortality. However, because we included other cardiovascular outcomes that were assessed in other studies, we were able to compare results for individual endpoints.

Finally, our results should be taken in the context of the population sample that was included, which was limited to an insured adult T2D population in Texas. Therefore, findings may not be generalizable outside of our institution or geographic coverage. Additionally, a known limitation of propensity score matching is that results are not generalizable to the patients who were excluded.

Despite these limitations, our results were generally consistent with those from other studies and provides useful insights that can be applied to similar institutions to help guide health care decision making.

\section{Conclusions}

Both SGLT-2is and GLP-1 RAs showed significant reductions in the composite cardiovascular outcome as well as unstable angina requiring hospitalization compared with oADMs. However, only SGLT-2is were associated with a lower risk of hospitalizations for heart failure. Findings suggest that SGLT2is and GLP-1 RAs are both equally effective at reducing cardiovascular events in patients with T2D compared with oADMs, whereas SGLT-2is may be more effective at reducing heart failure-related events. Nevertheless, cardiovascular outcomes were similar between SGLT-2is and GLP-1 RAs when compared with each other. This study provides realworld evidence for health care decision makers to consider the selection of these novel antidiabetic agents with demonstrated cardiovascular benefits over other agents regardless of CVD status. Future investigation is needed regarding whether heart failure benefit with SGLT-2is is similar between patients with reduced ejection fraction and those with preserved ejection fraction. Furthermore, investigation surrounding use of both drug classes in the absence of T2D for CVD risk reduction and in combination may be warranted, given their distinct mechanism of actions and cardiovascular benefits that are independent of their glycemic effects. 


\section{Authors}

ELMOR D. PINEDA, PharmD, MS, and PAUL J. GODLEY, PharmD, Department of Pharmacy, Baylor Scott \& White Health, Temple, Texas, and College of Pharmacy, University of Texas at Austin. I-CHIA LIAO, MPH, Center for Applied Health Research, Baylor Scott \& White Health, Temple, Texas; JEFFREY B. MICHEL, $M D$, Division of Cardiology, Baylor Scott and White Medical Center, Temple, Texas; and KAREN L. RASCATI, PhD, College of Pharmacy, University of Texas at Austin.

AUTHOR CORRESPONDENCE: Elmor D. Pineda, PharmD, MS, E-mail: Elmor.Pineda95@gmail.com.

\section{DISCLOSURES}

No outside funding supported this study. The authors have no conflicts of interest to report.

\section{REFERENCES}

1. Low Wang Cecilia C, Hess Connie N, Hiatt William R, Goldfine Allison B. Clinical update: cardiovascular disease in diabetes mellitus. Circulation. 2016;133(24):2459-502.

2. Booth GL, Kapral MK, Fung K, Tu JV. Relation between age and cardiovascular disease in men and women with diabetes compared with non-diabetic people: a population-based retrospective cohort study. Lancet. 2006;368(9529):29-36.

3. Kannel WB, McGee DL. Diabetes and cardiovascular disease: the Framingham study. JAMA. 1979;241(19):2035-38.

4. American Diabetes Association. Economic costs of diabetes in the U.S. in 2017. Diabetes Care. 2018;41(5):917-98

5. American Diabetes Association. Economic costs of diabetes in the U.S. in 2012. Diabetes Care. 2013;36(4):1033-46.
6. Einarson TR, Acs A, Ludwig C, Panton UH. Economic burden of cardiovascular disease in type 2 diabetes: a systematic review. Value Health. 2018;21(7):881-90.

7. Arnett DK, Blumenthal RS, Albert MA, et al. 2019 ACC/AHA Guideline on the primary prevention of cardiovascular disease. J Am Coll Cardiol. 2019;10;74(10):1376-414

8. Kosiborod M, Cavender MA, Fu AZ, et al. Lower risk of heart failure and death in patients initiated on sodium-glucose cotransporter-2 inhibitors versus other glucose-lowering drugs. Circulation. 2017;136(3):249-59.

9. Kosiborod M, Lam CSP, Kohsaka S, et al. Cardiovascular events associated with SGLT-2 inhibitors versus other glucose-lowering drugs: the CVDREAL 2 study. J Am Coll Cardiol. 2018;71(23):2628-39.

10. Patorno E, Goldfine AB, Schneeweiss S, et al. Cardiovascular outcomes associated with canagliflozin versus other non-gliflozin antidiabetic drugs: population based cohort study. BMJ. 2018;360:k119.

11. Lund JL, Richardson DB, Stürmer T. The active comparator, new user study design in pharmacoepidemiology: historical foundations and contemporary application. Curr Epidemiol Rep. 2015;2(4):221-28.

12. University of Manitoba. Concept: Charlson Comorbidity Index. January 17, 2019. Available at: http://mchp-appserv.cpe.umanitoba.ca/viewConcept. php?conceptID=1098. Accessed March 19, 2020.

13. Zinman B, Wanner C, Lachin JM, et al. Empagliflozin, cardiovascular outcomes, and mortality in type 2 diabetes. N Engl J Med. 2015;373(22):2117-28.

14. Neal B, Perkovic V, Mahaffey KW, et al. Canagliflozin and cardiovascular and renal events in type 2 diabetes. N Engl J Med. 2017;377(7):644-57.

15. Wiviott SD, Raz I, Bonaca MP, et al. Dapagliflozin and cardiovascular outcomes in type 2 diabetes. N Engl J Med. 2019;380(4):347-57.

16. Marx N, Libby P. Cardiovascular benefits of GLP-1 receptor agonism: is inflammation a key? JACC: Basic Transl Sci. 2018;3(6):858-60.

17. Choi J, Dekkers OM, le Cessie S. A comparison of different methods to handle missing data in the context of propensity score analysis. Eur J Epidemiol. 2019;34(1):23-36.

18. Fox CS, Sullivan L, D'Agostino RB, Wilson PWF. The significant effect of diabetes duration on coronary heart disease mortality: the Framingham heart study. Diabetes Care. 2004;27(3):704-08. 
Cardiovascular Outcomes Among Patients with Type 2 Diabetes Newly Initiated on Sodium-Glucose Cotransporter-2 Inhibitors, Glucagon-Like Peptide-1 Receptor Agonists, and Other Antidiabetic Medications

\begin{tabular}{|c|c|c|}
\hline APPENDIX A & $\begin{array}{l}\text { Diagnosi } \\
\text { Outcome }\end{array}$ & les for Cardiovascular \\
\hline Outcome & Code Type & Relevant Code \\
\hline Myocardial infarction & ICD-9-CM & 410.xx \\
\hline & ICD-10-CM & $\begin{array}{l}\text { I21.xx, I22.xx, I23.0, I23.1, } \\
\text { I23.2, I23.3, I23.4, I23.5, I23.6, } \\
\text { I23.8 }\end{array}$ \\
\hline & ICD-9-CM & 433.xl, 434.xl, 435.x, 436.x \\
\hline Stroke & ICD-10-CM & $\begin{array}{l}\text { G43.6xx, G45.0, G45.1, G45.2, } \\
\text { G45.8, G45.9, G46.1, G46.2, } \\
\text { G46.3, G46.4, G95.11, I63.xxx, } \\
\text { I67.84x, I97.8xxx }\end{array}$ \\
\hline Unstable angina & ICD-9-CM & 411.xx \\
\hline $\begin{array}{l}\text { requiring hospital- } \\
\text { ization }\end{array}$ & ICD-10-CM & $\begin{array}{l}\text { I20.0, I24.x, I25.110, I25.119, } \\
\text { I25.7x0, I25.7x9 }\end{array}$ \\
\hline Hospitalization for & ICD-9-CM & 402.xl, 404.xl, 404.x3, 428.x \\
\hline heart failure & ICD-10-CM & Il1.0, I13.0, I13.2, I50.xxx \\
\hline $\begin{array}{l}\text { Coronary revascular- } \\
\text { ization }\end{array}$ & ICD-9-CM & $\begin{array}{l}00.4,00.40,00.41,00.42 \\
00.43,00.44,00.45,00.46 \\
00.47,00.48,00.66,36.0 x \\
36.1 x\end{array}$ \\
\hline & ICD-10-CM & $\begin{array}{l}\text { 0210-0213, 0270-0273, } \\
\text { 02C0-02C3, 02CxxZZ, } \\
\text { 02QxxZZ, X2C0361, X2C1361, } \\
\text { X2C2361, X2C3361 }\end{array}$ \\
\hline & CPT-4 & $\begin{array}{l}33510-33516,33517-33536, \\
33572,4110 F, 92920-92944, \\
92973\end{array}$ \\
\hline
\end{tabular}

CPT-4 = Current Procedural Terminology, 4th Edition; ICD-9-CM = International Classification of Diseases, Ninth Revision, Clinical Modification; ICD-10-CM= International Classification of Diseases, Tenth Revision, Clinical Modification. 
Cardiovascular Outcomes Among Patients with Type 2 Diabetes Newly Initiated on Sodium-Glucose Cotransporter-2 Inhibitors, Glucagon-Like Peptide-1 Receptor Agonists, and Other Antidiabetic Medications

\begin{tabular}{|c|c|c|c|c|c|c|c|}
\hline \multirow[b]{2}{*}{ Variables } & \multicolumn{2}{|c|}{ Cohort 1} & \multicolumn{2}{|c|}{ Cohort 2} & \multicolumn{3}{|c|}{ Cohort 3} \\
\hline & SGLT-2i $(n=817)$ & oADM $(n=817)$ & GLP-1 RA $(n=815)$ & $\mathrm{oADM}(\mathrm{n}=815)$ & SGLT-2i $(n=947)$ & \multicolumn{2}{|c|}{ GLP-1 RA $(n=947)$} \\
\hline \multicolumn{8}{|c|}{ Index medications, n (\%) } \\
\hline AGI & $(0.0)$ & $(3.8)$ & $(0.0)$ & $(4.1)$ & $(0.0)$ & 0 & $(0.0)$ \\
\hline Metformin & $(0.0)$ & $137 \quad(16.8)$ & $(0.0)$ & $159 \quad(19.5)$ & $(0.0)$ & 0 & $(0.0)$ \\
\hline DPP-4i & $(0.0)$ & $144 \quad(17.6)$ & $(0.0)$ & $125 \quad(15.3)$ & $(0.0)$ & 0 & $(0.0)$ \\
\hline GLP-1 RA & $(0.0)$ & $(0.0)$ & $815 \quad(100.0)$ & $(0.0)$ & $(0.0)$ & 0 & $(0.0)$ \\
\hline SGLT-2i & $817 \quad(100.0)$ & $(0.0)$ & $(0.0)$ & $(0.0)$ & $947 \quad(100.0)$ & 0 & $(0.0)$ \\
\hline SU & $0 \quad(0.0)$ & $241 \quad(29.5)$ & $(0.0)$ & $257 \quad(31.5)$ & $\begin{array}{ll}0 & (0.0) \\
\end{array}$ & 0 & $(0.0)$ \\
\hline TZD & $(0.0)$ & $(8.8)$ & $(0.0)$ & $(7.6)$ & $(0.0)$ & 0 & $(0.0)$ \\
\hline Meglinitide & $(0.0)$ & $(0.2)$ & $(0.0)$ & $(0.3)$ & $(0.0)$ & 0 & $(0.0)$ \\
\hline Insulin & $(0.0)$ & $190 \quad(23.3)$ & $(0.0)$ & $190 \quad(23.3)$ & $(0.0)$ & 0 & $(0.0)$ \\
\hline
\end{tabular}

AGI = alpha-glucosidase inhibitor; DPP-4i=dipeptidyl peptidase-4 inhibitor; GLP-1 RA=glucagon-like peptide-1 receptor agonist; SGLT-2i=sodium-glucose cotransporter-2 inhibitor; $S U=$ sulfonylurea; TZD = thiazolidinedione. 